\title{
The inhibitory effects of recombinant plasminogen kringle 1-3 on the neovascularization of rabbit cornea induced by angiogenin, bFGF, and VEGF
}

\author{
Jung Hwan Kim ${ }^{1,4}$, Jae Chan Kim, \\ Seung Hwan Shin ${ }^{1}$, Soo-lk Chang ${ }^{2}$, \\ Hyo Sil Lee ${ }^{3}$ and Soo II Chung ${ }^{3}$ \\ ${ }^{1}$ Department of Ophthalmology, Chung-ang University Hospital, \\ Seoul, Korea \\ ${ }^{2}$ Department of Biochemistry, College of Natural Science, \\ Chung-buk University \\ ${ }^{3}$ Cheong-ju, Korea, Mog-am Biotechnology Research Institute, \\ Yong-in, Korea \\ ${ }^{4}$ Corresponding author: Tel, 82-2-748-9580; Fax, 82-2-3778-6491; \\ E-mail, cauheye@chollian.net
}

Accepted 2 December 1999

Abbrebiations: CAM, chorioallantoic membrane assay; $\mathrm{K} 1$, kringle 1; K2, kringle 2; K3, kringle 3; K4, kringle 4; rPK1-3, recombinant plasminogen kringle1-3; K1-4, kringle1-4; ARVO, The association for Research in Vision and Ophthalmology

\begin{abstract}
Angiostatin is a potent angiogenesis inhibitor that is composed of the first four kringles of plasminogen fragment. Angiostatin with one less kringle molecule (kringle 1 to 3) was recently demonstrated to be an effective angiogenic inhibitor. To determine whether recombinant plasminogen kringle 1-3 (rPK1-3) can inhibit the corneal neovascularization induced by potent angiogenic factors; angiogenin, bFGF, or VEGF, hydron polymer discs each containing $2.0 \mu \mathrm{g}$ of angiogenin, $500 \mathrm{ng}$ of bFGF, or $500 \mathrm{ng}$ of VEGF respectively were implanted into the corneal stroma of 138 rabbit eyes, and then discs each containing $10 \mu \mathrm{g}, 12.5 \mu \mathrm{g}, 20 \mu \mathrm{g}$ or $30 \mu \mathrm{g}$ of rPK1-3 were implanted randomly. Discs containing phosphate buffered saline were also implanted as a control. The angiogenesis score on number and length of newly formed vessels on the each of the rabbit's cornea were recorded daily by two observers (blinded). The treated corneas were also examined histologically. Recombinant PK1-3 treated corneas showed less neovascularization induced by all angiogenic factors $(p<0.05)$. and the extent of inhibition of neovascularization was proportional to the concentration of rPK1-3 ( $p<0.05)$. Histologic examination showed leukocyte infiltration into the corneal stroma on the
\end{abstract}

PBS treated eyes whereas rPK1-3 treated eyes showed only traces of leukocytes. These results of the effective rPK1-3 inhibition of corneal neovascularization induced by angiogenin, bFGF, or VEGF suggest that this angiostatin related fragment, rPK13 , may be useful in the treatment of various neovascular diseases.

Keywords: angiogenin, bFGF, corneal neovascularization, recombinant kringle 1-3 of plasminogen, VEGF

\section{Introduction}

Angiogenesis is essential in tissue reproduction, development, and wound repair. However, angiogenesis is also associated with pathogenesis of numerous eye diseases; loss of visual acuity, neovascular glaucoma, diabetic retinopathy, chemical burns and viral infections of the cornea (Epstein et al., 1987). Corneal neovascularization is also thought to predispose to the rejection of corneal allograft by facilitating the exposure of antigens to the host immune system (Hill and Maske, 1988). The mechanism of corneal neovascularization has been extensively investigated, in lieu of various mediators such as basic fibroblastic growth factor (bFGF) (Adamis et al., 1991), vascular endothelial growth factor (VEGF) (Amano et al., 1998), prostaglandins (Ziche et al., 1982), interleukin 2 and 8 (Koch et al., 1992; Lipman et al., 1992), and platelet derived endothelial cell growth factor (PD-ECGF) (Risau, 1990). Recently, a number of antiangiogenic molecules in the physiological system have been identified (Folkman, 1995). Angiostatin purified from serum and urine of mice bearing a murine Lewis lung carcinoma has been demonstrated to be one of the potent angiogenesis inhibitors specially that targets proliferating endothelial cells (O'Reilly et al., 1994; O'Reilly et al., 1996). Angiostatin contains the first four out of five repeating loop of plasminogen molecule known as the kringle domain. Each kringle is composed of 80 amino acids polypeptide chain linked by disulfide bonds at an end and at midregion forming two, an inner and an outer loop structures. In vitro, it specifically inhibits endothelial cell growth, but not proliferation of other cell types, including tumor cells. In vivo, angiostatin suppresses neovascularization in the chick chorioallantoic membrane assay (CAM) and the mouse corneal assay (O'Reilly et al., 1994; OReilly et al., 1996). Small fragments of angiostatin have been demonstrated to display differential 
effects on suppression of a growth of endothelial cell. The anti-proliferative activity of angiostatin on endothelial cell is shared by kringle 1 (K1), kringle 2 (K2), and kringle 3 (K3), but probably not by kringle 4 (K4). Slightly enhanced inhibition of BCE cell proliferation was reported when K4 was removed from angiostatin (Cao et al., 1996). The purpose of this study is to assess whether human rPK1-3 (Figure 1 diagram) affects the growth of new vessels in the rabbit cornea induced by angiogenin, bFGF, and VEGF.

\section{Materials and Methods}

\section{Manufacturing of recombinant kringle 1-3 of human plasminogen}

Construction, expression and purification of the rPK1-3 were carried out as follows; rPK1-3 (amino acid residues from 82 to 335 of human plasminogen) was amplified by polymerase chain reaction (PCR) using the following synthetic oligonucleotide primers on a template consisting of the cDNA of human plasminogen: (1) 5'primer: 5'CGGGATCCCATATGTCAG-AGTGCAAGACTGGGA-3',

(2) 3'-primer: 3'-CATTCTATGGCAGGACACTGAGGATCA-TTCCTAGGGC-5'. A Ndel site was included in the 5'-PCR primer. Bam $\mathrm{HI}$ site and stop codon (ATC, ATT) were incorporated into the 3-PCR primer. The cDNA encoding the $\mathrm{rK} 1-3$ was ligated into $\mathrm{Nde} \mathrm{I} / \mathrm{Bam} \mathrm{HI}$ site of the Escherichia coli (E. coli) expression vector, pET 11a, resulted in expression plasmids pMETK1. E.coli BL21 clone containing the highest number of the PMETK1 plasmid was selected and rK1-3 expression was induced by $1 \mathrm{mM}$ isopropyl thio- $\beta$-D-galactopyranoside. Cells were finally harvested by centrifugation for 30 minutes at $4,000 \times g$. The large-scale production of $\mathrm{rK} 1-3$, refolding and purification were carried out as follows: (1) The cells were disrupted by microfludizer. The insoluble body (IB) was collected by centrifugation and washed with lipophilic buffers until IB was 95\% pure; (2) The IB was then solubilized in $6 \mathrm{M}$ guanidine $\mathrm{HCl}$ containing $50 \mathrm{mM} \beta$ mercaptoethanol and refolding was accomplished in tris buffer, $\mathrm{pH}$ 8.6, containing basic amino acids and reducing agents; (3) The refolded rPK1-3 was purified to homogenicity by utilizing SP-Sepharose and Lysine-Sepharose columns (Pharmacia, NJ).

The analysis of its amino acid composition, N-terminal sequence, Circular Dichroism, and affinity toward 6-amino hexanoic acid-ligand of the purified rK1-3 showed that they were identical to those of the glycosylated native kringle 1-3 purified from the elastase digest of plasminogen. Furthermore, the immuno-reactivity against the monoclonal antibody to native $\mathrm{K} 1-4$ of plasminogen and biological properties measured by CAM assay, and bovine capillary endothelial cell proliferation assay in vitro showed comparable activities with those of the native PK1-3.
The purified rPK1-3 was stable during storage in lyophilized state at $4^{\circ} \mathrm{C}$ for more than a year.

\section{Chorioallantoic membrane assay}

Fertilized 3 day-old eggs were incubated at $37^{\circ} \mathrm{C}, 90 \%$ humidity. After 4.5 days of incubation, thermonox cover slips with bFGF + rK1-3 or bFGF + PBS (control) were applied on the CAM of each embryo. After 7 days of incubation, embryos and CAMs were analyzed for neovasculization with stereomicroscope.

\section{Preparation of pellet}

A rabbit corneal micropocket assay described by $\mathrm{Hu}$ et al. (1994) in detail was used. Briefly, $1 \mu \mathrm{g} / \mathrm{ml}$ of suspension of sterile phosphated buffered saline (Sigma, St. Louis, Mo) containing bovine angiogenin, recombinant bFGF (Scios Nova, Mountainview, CA), and human recombinant VEGF (Sigma, St. Louis, MO) respectively was added to $24 \%$ Hydron (polyhydroxyethyl-methacrylate [polyHEMA], Sigma, St. Louis, MO) in ethanol. A 4 $\mu$ aliquot of this mixture was then pipetted onto teflon pegs and allowed to dry under a laminar flow hood at room temperature. All procedures were performed under sterile condition. By this methods, each uniform pellet containing $2 \mu \mathrm{g}$ of bovine angiogenin, $500 \mathrm{ng}$ of bFGF, or $500 \mathrm{ng}$ of VEGF respectively was prepared. Also, hydron pellets containing $10,12.5,20$, or $30 \mu \mathrm{g}$ of rPK13 or PBS were prepared as described previously.

\section{Pellet implantation}

Each angiogenic factor-impregnated pellets (including angiogenin, bFGF, or VEGF) were implanted in 69 of New Zealand male white rabbits, weighing between 2.5 $\mathrm{kg}-3.0 \mathrm{~kg}$. All animals were handled and maintained in accordance with the ARVO Resolution on the Use of Animals in Ophthalmic and Vision Research. The animals were anesthetized by intramuscular injection of ketamine hydrochloride $(5 \mathrm{mg} / \mathrm{kg}$ ) and xylazine $(2 \mathrm{mg} / \mathrm{kg})$. The eyes were topically anesthetized with $0.5 \%$ propacaine (Ophthetic $^{\circledR}$, Alcon, TX), and the corneas were proptosed with a speculum. A intrastromal linear keratotomy (approximately $1.5 \mathrm{~mm}$ length) was performed under an operating microscope (Zeiss, Oberkochen ${ }^{\circledR}$, Germany) with a surgical blade (Bard-Parker no. 15; Becton Dickinson ${ }^{\circledR}$, Fraklin Lakes, NY) and lamellar micropocket was dissected with a $1.5 \mathrm{~mm}$ wide pliable iris spatula at $1.2 \mathrm{~mm}$ apart from the superior limbus. A hydrogel pellet containing $2 \mu \mathrm{g}$ of angiogenin was implanted in the micropocket located in the transparent corneal stroma of each 34 New Zealand white rabbit eyes. By the same manner, each hydrogel pellet containing $500 \mathrm{ng}$ of bFGF or $500 \mathrm{ng}$ of VEGF was implanted in the corneal stroma of the rabbit eyes (bFGF model; 40 eyes, VEGF model; 64 eyes). A second hydrogel pellet was placed in each eye adjacent to the first one, and then randomized to 
contain either rK1-3 or PBS (angiogenin model; pellets containing $12.5 \mathrm{mg}$ of $\mathrm{rK} 1-3$ in 20 eyes and PBS in 14 eyes, bFGF model; pellets containing $10 \mu \mathrm{g}$ of $\mathrm{rK} 1-3$ in 10 eye and $30 \mu \mathrm{g}$ of $\mathrm{rK} 1-3$ in 10 eyes and PBS in 20 eyes, VEGF model; pellets containing $10 \mu \mathrm{g}$ of $\mathrm{rK} 1-3$ in 10 eye, $20 \mu \mathrm{g}$ of rK1-3 in 12 eye and $30 \mu \mathrm{g}$ of rK1-3 in 10 eyes and PBS in 32 eyes). During the surgery, the cornea was irrigated with $0.5 \%$ chloramphenicol eye drops. On completion of the implantation, antibiotic eye ointment $\left(\right.$ Tobrex ${ }^{\circledR}$, Alcon, TX) was applied to the operated eye, not only to prevent infection but also to lubricate an irregular ocular surface.

\section{Biomicroscopic examination and score of angio- genesis}

The eyes were examined daily under slit lamp biomicroscopy by two blind observers. After rabbits were anesthetized with ketamine hydrochloride and xylazine, the eyes were proptosed. Pictures of corneal neovascularization were taken with zoom photographic slit lamp (model SM-50F; Takagi ${ }^{\circledR}$, Nakano, Japan). Corneal neovascularization was measured directly from slides using an image analyzer consisting of CCD camera (SONY ${ }^{\circledR}$ CCD TR-900, Japan) coupled with the digital analyzer system (Optima ${ }^{\circledR}$ version 5.1.1) on IBM compatible computer. Angiogenic activity was recorded and calculated by number of newly developed vessels multiplied by length of vessels from limbus on postoperative day 3 and 6 post pellet implantation. Length value of less than $0.3 \mathrm{~mm}$ was assigned to score $0 ; 1$, from $0.3 \mathrm{~mm}$ to $0.6 \mathrm{~mm}$; 2, from $0.7 \mathrm{~mm}$ to $0.9 \mathrm{~mm}$; and 3, for more than $1.0 \mathrm{~mm}$. In the case of a vessels branch into several vessels, a longest vessel was selected as a representative score. The scores of two blind-observer were summed and mean was used as final score. After recording the findings, the operated eyes were treated daily with $0.5 \%$ chloramphenicol eyedrops and Tobrex ${ }^{\circledR}$ ointment.

\section{Histological examination}

On post operation day 7, experimental animals were put into euthanasia by an air embolization after sedation with an intramuscular injection of ketamine hydrochloride. All of the eyes were enucleated immediately after euthanasia. Eyes enucleated on the 7th day of implantation were immersed in $4 \%$ paraformaldehyde solution, and then dissected under the operating microscope to make tissue blocks of $5 \times 5 \mathrm{~mm}$ in size. Each tissue block contained neovascularized cornea and upper limbus in full thickness. Slides were made with hematoxylin and eosin (H\&E) staining.

\section{Statistical analysis}

The data are reported as mean \pm standard deviation of experiments. Statistical analysis was performed by ana- lysis of variance followed by Mann-Whitney $U$ test to determine specific differences between two groups. $P$ value of $<0.05$ was considered statistically significant.

\section{Results}

\section{Chorioallantoic membrane assay}

After 3 days of the application of thermonox coverslips with bFGF and with or without rPK1-3 (Figure 1), embryos and CAMs were observed with stereomicroscope. An-giogenin and VEGF had also similar effects on the chorioallantoic membrane study. There was less visible vascularization in the areas covered with thermonox coverslips in the CAMs treated with rPK1-3. Whereas the control group showed prominent vascularization in those areas.

\section{Rabbit corneal study}

Biomicroscopic examination: Two pellets containing angiogenic factors and either angiostatin or PBS were implanted in rabbit corneas. Biomicroscopic observation revealed the healing process of the corneal epithelium and the stroma with mild edema within $24 \mathrm{~h}$ after surgery.

Angiogenin model: Two pellets containing bovine angiogenin and either rPK1-3 $(n=20)$ or PBS $(n=14)$ have been implanted in both groups. Limbal vessels began to develop toward the implant within a previously avascular cornea on postoperative day 2. And definite congestions of limbal blood vessel accompanied by mild angiogenic activity were observed in both groups. On the third day of post-implantation of angiogenin, the eyes treated with $\mathrm{rPK} 1-3$ had less angiogenesis (mean score $4.9 \pm 10.0$ ) than the eyes treated with PBS (mean

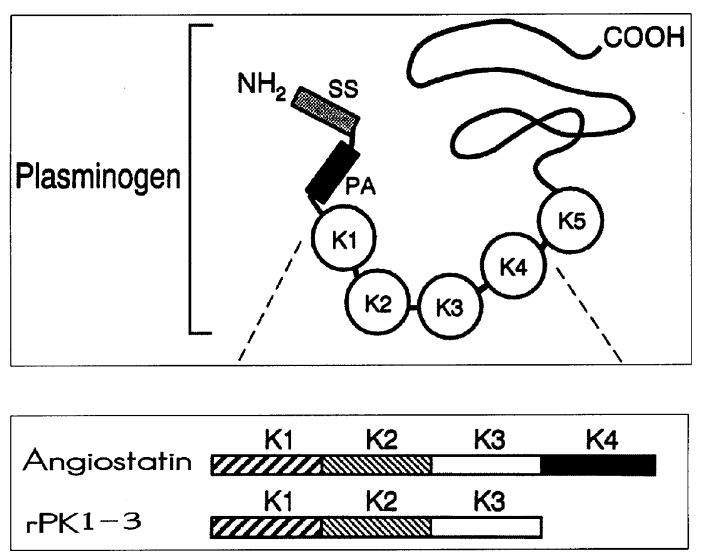

Figure 1. Schematic diagram of plasminogen (top) and angiostatin and rPK1-3 (bottom). Top graph shows a diagram of plasminogen where circles denote kringle 1-5 (k1, k2, K3, K5 \& K5) and the bottom horizontal bar graph shows angiostatin which is made up of serially linked kringles 1-4 and rPK13 with kringles 1-3. 
Corneal NV Score

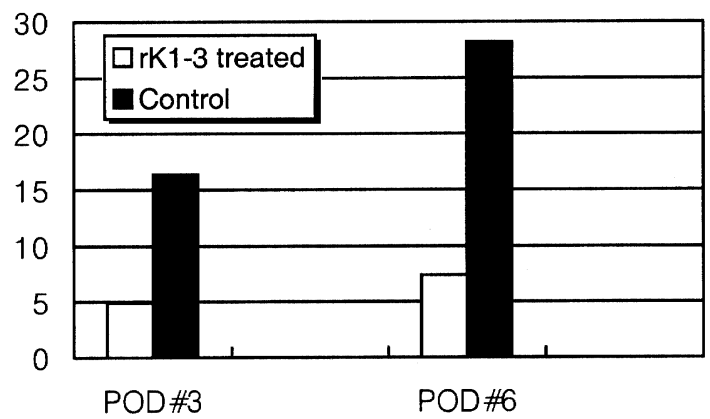

Figure 2. Inhibition of angiogenin-induced corneal neovascularization by recombinant kringle 1-3 (rPK1-3) ( $n=20$ eyes). Control animals received PBS ( $n=14$ eyes). Histogram shows mean angiogenesis score \pm standard deviation on post operative days 3 and 6

score $16.4 \pm 20.0)(p<0.05$, Figure 2$)$. On the sixth day, the eyes treated with rPK1-3 also had less angiogenesis (mean score $7.4 \pm 10.1$ ) than the eyes treated with PBS (mean score $28.2 \pm 42.7)(p<0.05$, Figure 2). As the vessels increased in length during this period of time, the extent of circumferential neovascularization stabilized by fifth to sixth day and decreased on the seventh day.

bFGF model: Two pellets containing bFGF and either rPK1-3 $(n=20)$ or PBS $(n=20)$ have been implanted in both groups. Limbal vessels began to develop toward the implant within a previously avascular cornea. Limbal hyperemias accompanied by angiogenic activity were observed in both groups. On the third day of postimplantation of bFGF, the eyes treated with $10 \mu \mathrm{g}$ and $30 \mu \mathrm{g}$ of $\mathrm{rPK} 1-3$ had less neovascularization than the eyes treated with PBS $(p<0.05$, Figure 3$)$. On the sixth day, the eyes treated with rPK1-3 also had less angiogenesis than the eyes treated with PBS $(p<0.05$, Figure 3). As the vessels increased in length during this period of time, the extent of circumferential neovascularization regressed on the seventh day after implantation. VEGF model: Two pellets containing VEGF and either

Corneal NV Score

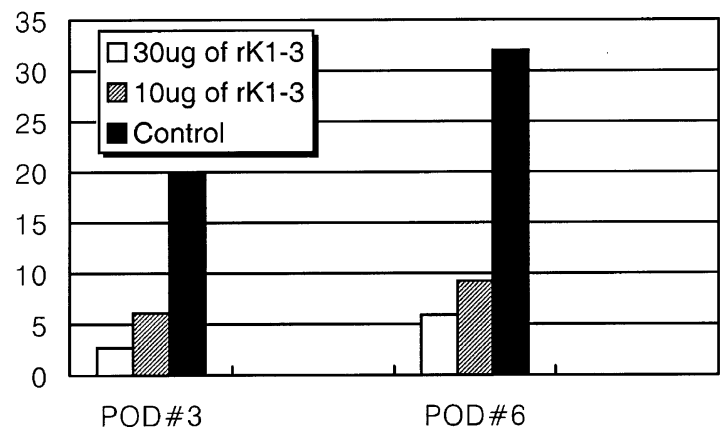

Figure 3. Recombinant kringle 1-3 (rPK1-3) treated eyes have significantly less angiogenesis score than PBS treated eyes in bFGF induced corneal neovascularization. Inhibitory activity of $r P K 1-3$ on corneal angiogenesis are proportional to its concentration.
Corneal NV Score

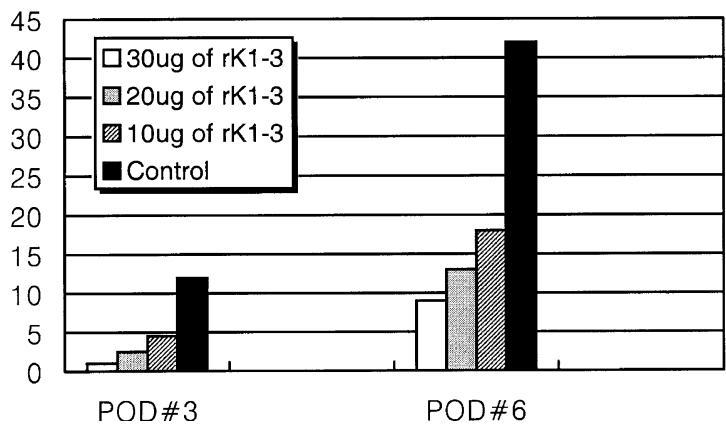

Figure 4. Recombinant kringle 1-3 (rPK1-3) treated eyes have significantly less angiogenesis score than PBS treated eyes in VEGF induced corneal neovascularization. Inhibitory activity of rPK1-3 on corneal angiogenesis are proportional to its concentration.

rPK1-3 ( $n=32)$ or PBS $(n=32)$ have been implanted in both groups. Prominent limbal hyperemias accompanied by angiogenic activity were observed in both groups. On the third day of post-implantation of VEGF, the eyes treated with rPK1-3 had less neovascularization than the eyes treated with PBS in all groups $(p<0.05$, Figure 4). On the sixth day, the eyes treated with rPK1-3 also had less angiogenesis than eye treated with PBS $(p<0.05$, Figure 4$)$. As the vessels increased in length
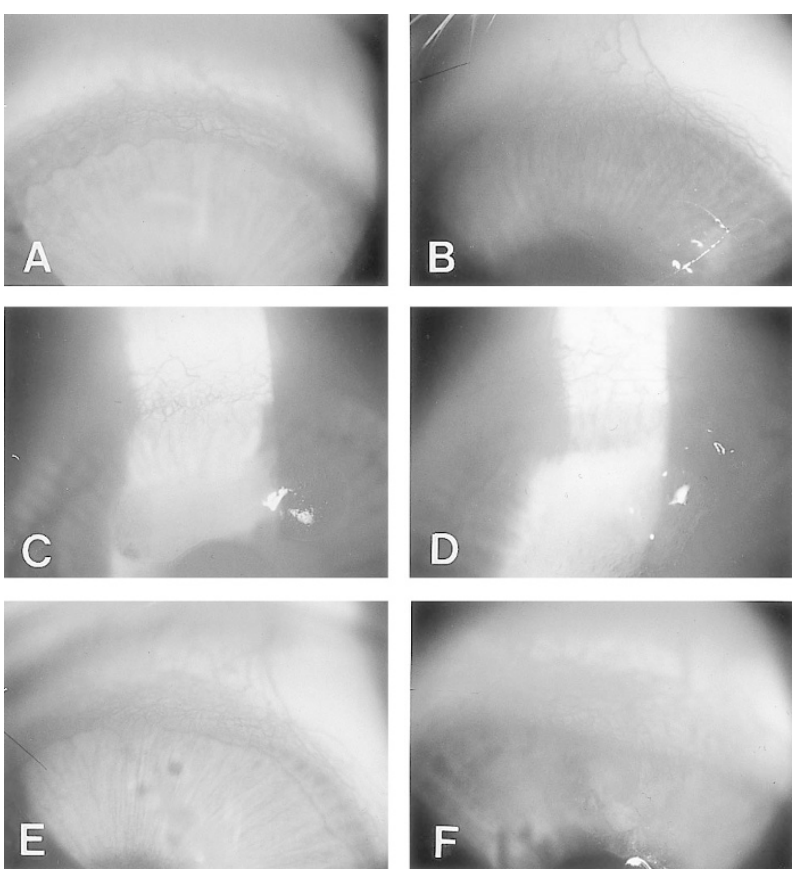

Figure 5. Slit lamp photographs of rabbit corneas 3 and 6 days after implantation show that recombinant kringle 1-3 (rPK1-3) treated eyes have less angiogenesis than PBS treated eyes. Angiogenin models; A: rPK1-3 treated eye, post operative day 6 . B: phosphated buffered saline (PBS) treated eye (control), postoperative day 6, bFGF models; C: rPK1-3 treated eye, post operative day 6. D: PBS (control) treated eye, post operative day 6. VEGF models; E: rPK1-3 treated eye, post operative day 6. F: PBS (control) treated eye, post operative day 6 . 


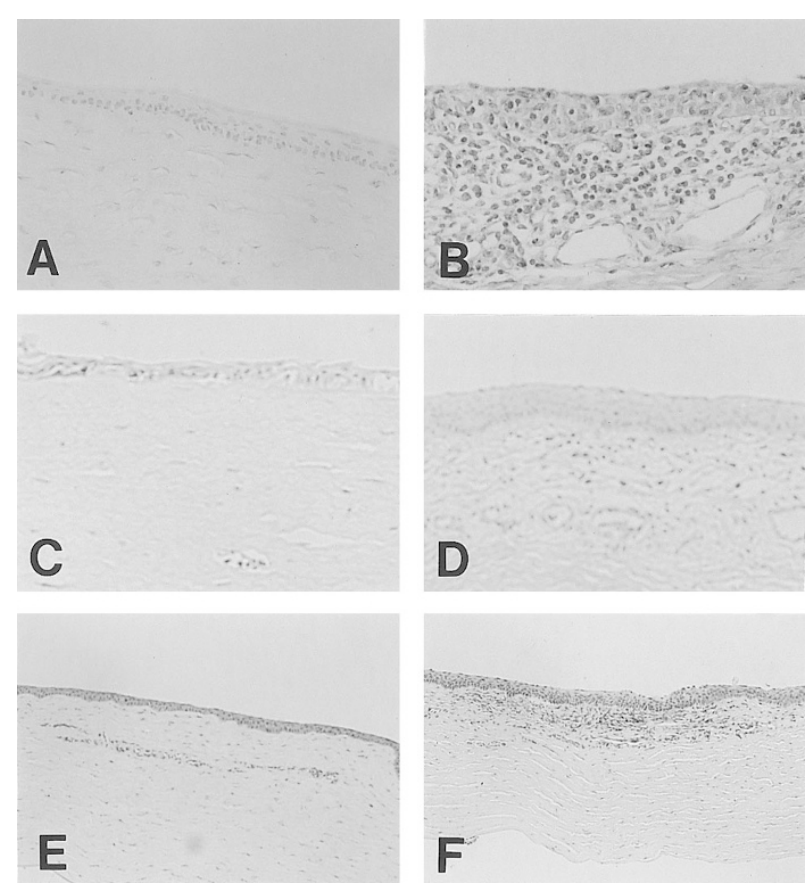

Figure 6. Histopathologic study reveals the control corneas treated with PBS have significantly more stromal leukocyte infiltration than rPK1-3 treated corneas at postopertive 6th day in all models (H\&E stain, x40). A. rPK1-3 treated cornea in angiogenin model, B. PBS treated cornea in angiogenin model, $\mathrm{C}$. rPK1-3 treated cornea in bFGF model, D. PBS treated cornea in bFGF model, E. rPK1-3 treated cornea in VEGF model, F. PBS treated cornea in VEGF model.

during this period of time, the extent of circumferential neovascularization regressed on the seventh day after implantation.

Histological examination: In the cornea of H\&E stained section, there were significantly more leukocyte infiltration in PBS treated group. Preceding the burst of the new vessel in the group treated with PBS, extensive influx of cellular infiltrates composed mainly of polymorphonuclear cells, lymphocytes, and macrophages were observed (Figure 6).

\section{Discussion}

Neovascularization affects several disease of the eye and results in loss of visual acuity. These diseases include neovascular glaucoma, diabetic retinopathy, chemical burns and viral infections of the cornea (Epstein et al., 1987). Corneal neovascularization is also thought to predispose to the rejection of corneal allografts by facilitating the exposure of antigens in donor cornea to the immune system (Hill and Maske, 1988). A variety of angiogenic factors can stimulate angiogenesis in vitro and vivo. This includes angiogenin (Fett et al., 1985; Bond and Vallee, 1988; Risau, 1990), basic fibroblastic growth factor (bFGF) (Adamis et al., 1991), vascular endothelial growth factor (VEGF) (Amano et al., 1998), prostaglandins (Ziche et al., 1982), interleukin 2 and 8 (Koch et al., 1992; Lipman et al., 1992), and platelet derived endothelial cell growth factor (PD-ECGF) (Risau, 1990). And also a number of antiangiogenic molecules have been identified (Folkman et al., 1995). Angiogenin is unique among the established angiogenic proteins (Schott and Marrow, 1993) in that it is isolated based solely on its ability to induce neovascularization in chick embryo chorioallantoic membrane (CAM) (Loughnan et al., 1996). It also induces blood vessel formation in the cornea and meniscus of the knee of the rabbit (King and Vallee, 1991). The activity of angiogenin, as other angiogenic proteins, can also be regulated by cellular mechanism (Badet et al., 1989). In vitro experiment, it has shown to stimulate endothelial cells in order to produce most of the characteristics of new capillary formation, including activation of proteases that digest the basement membrane and extracellular matrix $(\mathrm{Hu}$ et al., 1994), induction of cell adhesion (Soncin, 1992), and invasion (Hu et al., 1994) as well as organization of cells into tubules (Jim et al., 1995). Basic fibroblast growth factor is a multifunctional, single-chain peptide composed of 146 aminoacids (Esch et al., 1985) that has important effects on cell morphology, transformation, proliferation, and differentiation in vitro (Gospodarowicz et al., 1987). It stimulates chemotaxis in vascular endothelial cells and is a potent mitogen for mesoderm-derived cells in vitro (Terranova et al., 1985). Moreover, it induces capillary endothelial cells to invade a three-dimensional collagen matrix, to organize into tubules that resemble blood capillaries, and to produce plasminogen activator and proteases in vitro (Montesano et al., 1986). Because bFGF is capable of directly inducing series of steps required for angiogenesis, it is an appropriate choice to use in model system. Vascular endothelial growth factor (VEGF) is a heparin-binding angiogenic factor and is highly conserved, multifunctional cytokine expressed and secreted at high levels by activated macrophages and many tumor cells of animal and human origin (Senger et al., 1993). Among the various angiogenic factors identified to date, VEGF is unique because it specifically acts on endothelial cells (Connolly, 1991) and its cell-surface receptors are exclusively expressed in endothelial cells (Plate et al., 1993). The overexpression of VEGF and its receptors are associated with chronic inflammation, tumor growth and other angiogenic diseases. Thus, VEGF is a prime target for the development of specific angiosuppressive drugs recently. A number of angiogenesis inhibitors have been characterized as proteolytic fragments. Proteolytic processing plays critical dual roles in the control of angiogenesis. When angiogenesis is initiated, proteolytic degradation of the basement membrane surrounding endothelial cells is a prerequisite for endothelial cell proliferation. Once new blood vessels have been formed, they may require angiogenic inhibitors, such as the proteolytic fragments, to control the over- 
growth of blood vessels. Tumors not only overexpress angiogenic factors, but also retain their abilities of producing angiogenesis inhibitors. The switch to the angiogenic phenotype requires both up-regulation of angiogenic stimulators and down-regulation of angiogenesis inhibitors (Cao et al., 1996). It is not yet clear how expression of these angiogenesis inhibitors is regulated. However some tumors produce proteases that subsequently cleave circulating plasminogen to generate angiostatin, others may use different strategies for generation of angiostatin.

Angiostatin is a circulating angiogenesis inhibitor that has been purified from serum and urine of mice bearing a murine Lewis lung carcinoma (O'Reilly et al., 1994; O'Reilly et al., 1996). Angiostatin has been characterized as a potent inhibitor of angiogenesis which specially targets proliferating endothelial cells (Cao et al., 1996). Angiostatin is a $38 \mathrm{kD}$ internal fragment of plasminogen containing the first four kringle structures, which is known as the most effective inhibitor of tumor growth, as well as endogenous and synthetic angiogenesis inhibitors (Cao et al., 1996). Recombinant kringle 1 and kringle 3 show a potent inhibitory activity in the endothelial cell proliferation assay in vitro. Also recombinant kringle 2 displays a with lesser degree of inhibition. In contrast, kringle 4 is an ineffective inhibitor of bFGF-stimulated endothelial cell proliferation. Among the tandem kringle arrays, the most potent inhibitory activity is seen in the kringle 1-3 fragment compared to others (Cao et al., 1996). According to a recent report, kringle 5 of plasminogen also showed an antiproliferative activity on the endothelial cell (Cao et al., 1997) and inhibits endothelial cell migration (Ji et al., 1998).

The molecular mechanism underlying how angiostatin and its related kringle fragment specifically inhibit endothelial cell growth is unclear. Knowledge of this mech anism by which angiostatin and its related kringle fragments inhibit neovascularization is crucial to an understanding of its function in vivo and angiogenesis in general. Whether these inhibitors are angiostatin or angiostatinrelated molecule remains to be investigated. Recent studies demonstrated that angiostatin induces apoptosis in endothelial cells (Claesson-Welsh et al., 1998; Lucas et al., 1998) and also inhibits migration and tube formation of endothelial cells (Luo et al., 1998). Angiostatin selectively upregulates E-selectin in proliferating endothelial cells in vitro. This selectivity may provide insights into the mechanism by which angiostatin inhibits angiogenesis in vivo without apparent effects on quiescent endothelium (Redlitz et al., 1999). Ji et al. (1998) reported that kringle domains of angiostatin may contribute to overall antiangiogenic function by their distinct anti-migratory activities. Redlitz et al. (1999) demonstrated that angiostatin inhibits primarily the invasion of endothelial cells and exerts minimal effects on their proliferation.

Previous studies have shown that an inflammatory process played a major role in the neovascular response with bFGF (Loughnan et al., 1996). In this study, histolopathogic findings demonstrated that there was an intrastromal vascularization with many inflammatory cell infiltration on cornea implanted with pellets containing angiogenic factors and PBS. Less inflammatory cells were observed in rabbits implanted with pellets containing angiogenic factors and recombinant kringle 1-3 (rPK1$3)$. Thus, these results suggest that an inflammatory process plays an important role in the development of neovascularization by angiogenin, bFGF, and VEGF. These also suggest that angiostatin and its kringle structures may suppress inflammatory reaction in the angiogenesis process.

In this study, the eyes treated with recombinant kringle 1-3 showed less induction of corneal neovascularization than PBS treated control eyes in all angiogenic factor models $(p<0.05)$. And the extent of inhibition of neovascularization was proportional to the concentration of recombinant kringle 1-3 $(p<0.05)$. These findings provide a direct evidence that neovascularization induced by angiogenin, bFGF, and VEGF is inhibited by rPK1-3 in vivo. Based on our in vivo studies of the rabbit cornea micropocket assay, it is apparent that intrastromally implanted rPK1-3 is an inhibitor of angiogenesis induced by angiogenin, bFGF, or VEGF. Leukocyte infiltration has an important role in neovascularization induced by angiogenin, bFGF, and VEGF and angiostatin may suppress these processes. This study provides the direct evidence that neovascularization by angiogenin is inhibited by rPK1-3 in vivo. There were no noticable physiological ill effect during the course of this study. Therapeutic use of endogenous inhibitors is less likely to cause side effects such as suppression of hematopoiesis and gastrointestinal symptoms (Folkman J. 1995). Thus, rPK13 , endothelial cell-specific inhibitor such as angiostatin related fragment, may be useful for long term therapy in suppression of angiogenesis.

\section{References}

Adamis, A. P., Meklir, B. and Joyce, N. C. (1991) In situ injuryinduced release of basic-fibroblast growth factor from corneal endothelial cells. Am J pathol 139: 961-6

Amano, S., Rohan, R., Kuroki, M., Tolentino, M. and Adamis, A. P. (1998) Requirement for vascular endothelial growth factor in wound- and inflammation- related corneal neovascularization. Invet Ophthalmol Vis Sci 39: 18-22

Badet, J., Soncin, F., Guitton, J. D., Lamare, O., Cartwright, T. and Barritault, D. (1989) Angiogenin promotes invasiveness of cultured endothelial cells by stimulation of cell. Proc Natl Acad Sci USA 86: 8427-8431

Bond, M. D. and Vallee, B. L. (1988) Isolation of bovine angiogenin using a placental ribonuclease inhibitor binding assay. Biochemistry 27: 6282-7 
Cao, Y., Ji, R. W., Davidson, D., Schaller, J., Marti, D., Sohndel, S., McCanece, S. G., O'Reilly, M. S., Llinas, M. and Folkman, J. (1996) Kringle domain of human angiostatin, characterization of the anti-proliferative activity on endothelial cells. J Biol Chem 271: 29461-29467

Cao, Y., Chen, A., An, S. S. A., Ji, R. W., Davidson, D., Cao, Y. and Llinas, M. (1997) Kringle 5 of plasminogen is a novel inhibitor of endothelial cell growth. J Biol Chem. 36: 2292422928.

Claesson-Welsh, L., Welsh, M., Ito, N., Anand-Apte, B., Soker, S., Zetter, B., O'Reilly, M. and Folkman, J. (1998) Angiostatin induces endothelial cell apoptosis and activation of focal adhesion kinase independently of the integrin-binding motif RGD. Proc Natl Acd Sci USA 95(10): 5579-5583

Connolly, D. T. (1991) Vascular permeability factor: a unique regulator of blood vessel function. J cell Biochem. 47: 219-223

Epstein, R. J., Stulting, R. D., Hendrick, R. L. and Harris, D. M. (1987) Corneal neovascularization: pathogenesis and inhibition. Cornea 6: 250-8.

Esch, F., Baird, A. and Ling, N. (1985) Primay structure of bovine pituitary basic fibroblast growth factor (FGF) and comparison with the amino terminal sequence of bovine brain acidic FGF. Proc Natl Acad Sci USA 82: 6507-6511

Fett, J. W., Strydom, D. J., Lobb, R. R., Alderman, E. M., Bethune, J. L., Riordan, J. F. and Vallee, B. L. (1985) Isolation and characterization of angiogenin, an angiogenic protein from human carcinoma cells. Biochemistry 24: 5480-5486

Folkman, J. (1995) Seminars in medicine of the Beth Israel Hopital, Boston, clinical application of research on angiogenesis. N. Engl. J. Med. 333: 1757-1763

Gospodarowicz, D., Ferrara, N., Schweigerer, L. and Neufeld, G. (1987) Structural characterization and biological functions of fibroblast growth factor. Endocr Rev. 8(2): 95-110

Hill, J. C. and Maske, R. (1988) An animal model for corneal graft rejection in high-risk keratoplasty. Transplantation 46: 26 30

Hu, G., Riordan, J. F. and Valle, B. L. (1994) Angigenin promotes invasiveness of cultured endothelial cells by stimulation of cell. Proc Natl Acad Sci USA 91: 12096-12100.

Ji, W. R., Castellino, F. J., Chang, Y., Deford, M. E., Gray, H., Villarreal, X., Kondri, M. E., Marti, D. N., Llinas, M., Schaller, J., Kramer, R. A. and Trail, P. A. (1998) Characterization of kringle domains of angiostatin as antagonists of endothelial cell migration, an important process in angiogenesis. FASEB J. 15: $1731-8$

Jim, S. I., Ito, K. I., Kohno, K., Ono, M., Kuwano, M., Itagaki, Y. and Isikawa, H. (1995) Biochem. Biophys Res Commun. 211: $476-483$

King, T. V. and Vallee, B. L. J. (1991) Neovascularisation of the meniscus with angiogenin. An experimental study in rabbits. Bone Joint Surg 73-B: 587-590

Koch, A. E., Polverini, P. J., Kunkel, S. L., Harlow, L. A., Di Pietro, L. A., Elner, V. M., Elner, S. G. and Strieter, R. M. (1992) Interleukin-8 as a macrophage-derived mediator of angiogenensis. Science 2581798-801

Lipman, R. M., Epstein, R. J. and Hendricks, R. L. (1992) Suppression of corneal neovascularization with cyclosporin. Arch Ophthalmol. 110: 405-7

Loughnan, M. S., Chatzistefanou, K., Gonzalez, E. M., Flynn, E., Adamis, A. P., Shing, Y., DAmato, R. J. and Folkman, J. (1996) Experimental corneal neovascularisation using sucralfate and basic fibroblast factor. Aust N Z J Ophthalmol. 24: $289-295$

Lucas, R., Holmgren, L., Garcia, I., Jimenez, B., Mandriota, S. J., Borlat, F., Sim, B. K., Wu, Z., Grau, G. E., Shing, Y., Soff, G. A., Bouck, N. and Pepper, M. S. (1998) Multiple forms of angiostatin induce apoptosis in endothelial cells. Blood 92: 4730-4741

Luo, J., Lin, J., Paranya, G. and Bischoff, J. (1998) Angiostatin upregulates $\mathrm{E}$-selectin in proliferating endothelial cells. Biochem Biophys Res Commun. 243(3): 906-911

Montesano, R., Vassali, J. D., Baird, A., Gullemin, R. and Orci, L. (1986) Basic fibroblast growth factor induces angiogenesis in vitro. Proc Natl Acad Sci USA 83: 7297-7301

O’Reilly, M. S., Holmgren, L., Shing, Y., Chen, C., Rosenthal, R. A., Moses, M., Lane, W. S., Cao, Y., Sage, E. H. and Folkman, J. (1994) Angiostatin : a novel angiogenesis inhibitor that mediates the suppression of metastases by a Lewis lung carcinoma. Cell 79: 315-328

O’Reilly, M. S., Holmgren, L., Chen, C. and Folkman, J. (1996) Angiostatin induces and sustains dormancy of human primary tumor in mice. Nat. Med. 2: 689-692

Plate, K. H., Breier, G. H. A., Millauer, B., Ullrich, A. and Risau, W. (1993) Up-regulation of vascular endothelial growth factor and its cognate receptors in a rat glioma model of tumor angiogenesis. Cancer Res. 53: 5822-5827

Redlitz, A., Daum, G. and Sage, E. H. (1999) Angiostatin diminishes activation of the mitogen-activated protein kinases ERK-1 and ERK-2 in human dermal microvascular endothelial cells. J Vasc Res. 36(1): 28-

Risau, W. (1990) Angiogenic growth factors. Prog Growth Factor Res 2: 71-9

Schott, R. J. and Marrow, L. A. (1993) Growth factors and angiogenesis. Cardiovasc. Res. 27: 1155-1161.

Senger, D. R., Water, L. V. D., Brown, L. F., Nagy, J. A., Yeo, N. K. T., Yeo, T. K., Berse, B., Jackman, R. W., Dvorak, A. M. and Dvorak, H. F. (1993) Vascular permeability factor (VPF, VEGF) in tumor biology. Cancer Metastasis Rev. 12: 303-324

Soncin, F. (1992) Angiogenin supports endothelial and fibroblast cell adhesion associated proteolytic activities. Proc Natl Acad Sci USA 89: 2232-2236.

Terranova, V. P., DiFlorio, R., Lyall, R. M., Hic, S., Friesel, R. and Maciag T. (1985) Human endothelial cells are chemotactic to endothelial growth factor and heparin. J Cell Biol. 101: 2330-2334.

Ziche, M., Jones, J. and Gullino, P. M. (1982) Role of prostaglandin E1 and copper in angiogenesis. J Natl Cancer Inst 69: 475-80. 